\title{
Comparative Gene Expression Analysis of Lymphocytes Treated with Exosomes Derived from Ovarian Cancer and Ovarian Cysts
}

\author{
Yujuan $\mathrm{Li}^{1+}$, Yang Yang ${ }^{1+}$, Aiwei Xiong ${ }^{1}$, Xiaoqin $\mathrm{Wu}^{1}$, Jingyan Xie', Suping Han ${ }^{1}$ \\ and Shuli Zhao ${ }^{1,2 *}$ \\ ${ }^{1}$ Nanjing First Hospital, Nanjing Medical University, Nanjing, China, ${ }^{2}$ State Key Laboratory of Reproductive Medicine, \\ Nanjing Medical University, Nanjing, China
}

\section{OPEN ACCESS}

Edited by:

Sherven Sharma,

VA Greater Los Angeles

Healthcare System (VHA),

United States

Reviewed by:

Magdalena Plebanski,

Monash University, Australia

Carlo Pucillo,

University of Udine, Italy

*Correspondence:

Shuli Zhao

shulizhao79@163.com

tThese authors have contributed equally to this work.

Specialty section:

This article was submitted to

Cancer Immunity and

Immunotherapy,

a section of the journal

Frontiers in Immunology

Received: 07 September 2016

Accepted: 08 May 2017

Published: 01 June 2017

Citation:

Li Y, Yang Y, Xiong A, Wu X, Xie J, Han S and Zhao S (2017)

Comparative Gene Expression

Analysis of Lymphocytes Treated with

Exosomes Derived from Ovarian

Cancer and Ovarian Cysts.

Front. Immunol. 8:607.

doi: 10.3389/fimmu.2017.00607
Cancer cells employ many strategies to evade immune defense and to facilitate tumor growth and angiogenesis. As a novel mode of intercellular communication, cancerderived exosomes contribute to the recruitment and mediation of lymphocytes within the tumor environment. However, the mechanisms and key molecules mediating the effect of exosomes on lymphocytes are unclear. We treated healthy peripheral blood lymphocytes with exosomes from ovarian cancer and ovarian cysts and screened for differentially expressed genes using the $\mathrm{RT}^{2}$ Profiler Cancer Inflammation and Immunity Crosstalk PCR Array. A total of 26 upregulated genes (mainly pro-inflammatory genes and immunostimulatory and immunosuppressive factor) and two downregulated genes (antigen presentation HLA-A/B) were identified. Western blotting using lymphocytes from malignant ascites and peritoneal washings of benign ovarian cysts suggested that the interferon and NF-kB signaling pathway were involved in the immune regulation of malignant exosomes. Out of 28 differentially expressed genes detected using the array, 11 were validated by real-time PCR using lymphocytes within ovarian cancer $(n=27)$ and ovarian cyst $(n=9)$ environments. In conclusion, our findings indicate that malignant cells secrete exosomes in the tumor microenvironment to recruit lymphocytes in order to suppress antitumor immunity (IL10, Foxp3, and HLA-A/B) and enhance tumor invasion, angiogenesis, and dissemination of proinflammatory cytokines (such as IL6 and VEGFA) via the interferon and NF- $\mathrm{kB}$ signaling pathways. These results clarify lymphocyte-cancer cell cross talk via exosomes and may facilitate the development of effective immunotherapeutic strategies for ovarian cancer.

Keywords: exosomes, ovarian cancer, ovarian cysts, lymphocytes, tumor microenvironment

\section{INTRODUCTION}

Ovarian cancer is the fourth most common female cancer worldwide and has the highest mortality rate of all gynecologic cancers in China $(1,2)$. Many studies have demonstrated that the tumor cell microenvironment plays an important role in ovarian cancer progression (3-5). However, the precise roles of microenvironment-tumor interactions are unclear, including the key substances in the microenvironment and the mechanisms that trigger tumor development, angiogenesis, and 
metastasis. Recently, many studies have reported that exosomes in the microenvironment, which are secreted by most normal and malignant cells, play critical roles in the occurrence and development of cancer (6-9).

As a novel mode of intercellular communication, exosomes can shuttle between cells and transmit signals by transporting various bioactive proteins and nucleic acids (e.g., DNA, mRNA, and miRNA) $(10,11)$. Andre et al. reported that exosomes isolated from ovarian cancer ascites contain antigen-presenting molecules, tetraspanins (CD81), and tumor antigens (Her2/Neu, Mart1, TRP, and gp100) (12). Growth factors, including tumor necrosis factor-alpha (TNF- $\alpha$ ), epidermal growth factor, and fibroblast growth factor, as well as mRNAs and miRNAs have been detected in exosomes (13). To date, 4,563 proteins, 1,639 mRNAs, and 764 miRNAs have been found in exosomes (14).

As a permissive reactive tumor-host microenvironment, malignant ascites provide sustenance for the growth and progression of ovarian cancer, including cytokines, chemokines, growth factors, and so on $(15,16)$. Our previous studies have demonstrated that immune cell subsets and the expression levels of miRNAs (17) that regulate the differentiation and development of dendritic cells in ascites differ from those in the peripheral blood of ovarian cancer patients (18), and these differences can result in the immune escape of tumor cells. Similarly, the same amenities in ascites could help tumor cells evade host immunosurveillance, enabling unlimited tumor cell growth $(19,20)$. However, the causal factors in ascites and specific mechanisms are poorly understood. Exosomes may play an important role and accordingly have been a focus of recent research $(21,22)$. In this study, the mRNA profiles in peripheral blood lymphocytes (PBLs) stimulated by exosomes derived from benign or malignant ascites were examined using the $\mathrm{RT}^{2}$ Profiler PCR Array and the key effectors of exosomes with respect to the immune system were explored.

\section{MATERIALS AND METHODS}

\section{Subjects}

In total, six epithelial ovarian cancer (EOC) patients and six benign ovarian cyst patients were recruited from the Department of Gynecology at Nanjing First Hospital, Nanjing Medical University (Nanjing, China). The diagnosis of EOC and benign ovarian cysts were in accordance with recommended criteria proposed by the World Health Organization in 2004. In addition, five age- and gender-matched healthy volunteers were recruited as healthy control. Sterile ascites from EOC patients or peritoneal washings from ovarian cysts were collected in sterilized 50-mL centrifuge tubes during surgery prior to therapeutic treatment. For isolation of peripheral lymphocytes, fasting venous blood samples $(10 \mathrm{~mL})$ from each healthy volunteer were collected by vein puncture using. All research involving human participants was conducted with approval from the Ethics Committee of Nanjing Medical University. Written informed consent was obtained from all participants. None of the patients were treated with preoperative chemotherapy or radiation.

\section{Isolation and Quantification of Exosomes}

Exosomes from all samples of malignant ascites or peritoneal washings were prepared using Total Exosome Isolation (from body fluids) (Catalog Number: 4484453, Invitrogen Inc., Carlsbad, CA, USA) according to the manufacturer's instructions, with some modifications (23). Briefly, all samples in sterilized 50 - $\mathrm{mL}$ centrifuge tubes were centrifuged at $2,000 \times g$ for $30 \mathrm{~min}$ to remove floating cells and debris. Then, supernatants were thoroughly mixed with 0.5 volumes of Total Exosome Isolation Reagent (from other body fluids) by pipetting repeatedly until the solution was homogenous, and samples were incubated at room temperature for $30 \mathrm{~min}$. After incubation, the mixed solutions were centrifuged at $10,000 \times g$ at room temperature for 10 min (XE-90; Beckman Coulter, Brea, CA, USA). The exosome pellets were washed and re-suspended in $100 \mu \mathrm{L}$ of phosphatebuffered saline (PBS) for quantification using a CD63 exoELISA (catalog\# EXOEL-CD63A-1; System Biosciences, Mountain View, CA, USA) according to the manufacturer's instructions (24). Finally, the purified exosomes were re-suspended in PBS at a concentration of $\sim 10^{10}$ exosome particles per milliliter and stored at $-80^{\circ} \mathrm{C}$ until use.

\section{Electron Microscopy}

Transmission electron microscopy was used to evaluate the morphology of isolated exosomes (25). Briefly, the exosome pellets were fixed in $5 \%$ glutaraldehyde for $2 \mathrm{~h}$ at $4^{\circ} \mathrm{C}$, and were washed with phosphoric acid buffer four times for $15 \mathrm{~min}$ each. After $2 \mathrm{~h}$ of fixation by $1 \%$ osmic acid at $4^{\circ} \mathrm{C}$, the adsorbed exosomes were washed with phosphoric acid buffer two times for $5 \mathrm{~min}$ each. Then, the prepared exosomes were stained with $2 \%$ uranyl acetate for $2 \mathrm{~h}$ and dehydrated in acetone. After they were embedded in embedding agent, the exosomes were sectioned at a thickness of $50-80 \mathrm{~nm}$ on an ultramicrotome. Finally, the prepared exosomes were observed under a transmission electron microscope (JEM-1010; JEOL, Tokyo, Japan) at $80.0 \mathrm{kV}$, and images were captured using a digital camera.

\section{Coincubation of PBLs with Exosomes}

Peripheral blood lymphocytes from five aged-matched healthy female volunteers were isolated using lymphocyte separation medium (TBDscience, Tianjin, China) density gradient centrifugation according to the manufacturer's instructions. Then, PBLs were purified using anti-CD45-PE antibody fluorescence-activated cell sorting performed on a Coulter Epics Elite ESP Flow Cytometer (Coulter, Brea, CA, USA). Subsequently, sorted PBLs were cultured in 6-well plates at $1 \times 10^{6}$ cells/well in triplicate using the AIM-V-free serum medium CTS (Gibco, Waltham, MA, USA). Isolated exosomes (three malignant exosomes or three benign exosomes, $1 \times 10^{9}$ exosome particles per milliter concentration) were added to each well, respectively. The cells were cultured for $48 \mathrm{~h}$ at $37^{\circ} \mathrm{C}$. Following extensive washing in PBS to remove exosomes, all PBLs in every group were pooled to isolate RNA.

\section{RNA Extraction and cDNA Preparation}

After coincubation with different exosomes, PBLs were collected to extract total RNA using the E.Z.N.A. ${ }^{\circledR}$ Total RNA 
Kit II (OMEGA, Biel, Switzerland, USA) according to the manufacturer's instructions. Next, the cDNA was synthesized using the $\mathrm{RT}^{2}$ First Strand Kit (Qiagen, Valencia, CA, USA) following the manufacturer's instructions.

\section{$\mathbf{R T}^{2}$ Profiler $^{\mathrm{TM}}$ PCR Array}

Eighty-four genes or biological pathways involved in mediating communication between tumor cells and the cellular mediators of inflammation and immunity were analyzed using the $\mathrm{RT}^{2}$ Profiler Cancer Inflammation and Immunity Crosstalk PCR Array (PAHS-181Z; SABiosciences, Frederick, MD, USA) (26). According to the manufacturer's protocol, real-time PCR was performed using $\mathrm{RT}^{2}$ Profiler PCR Arrays in combination with RT2 SYBR Green/ROX PCR Master Mix (Qiagen). A $102-\mu \mathrm{L}$ cDNA synthesis reaction volume was mixed with $2 \times$ RT2 SYBR Green Mastermix and RNase-free water to obtain a total volume of $2,700 \mu \mathrm{L}$. Subsequently, $25 \mu \mathrm{L}$ of the PCR component mix was placed into each well of the PCR array (a 96-well array). The three steps of the cycling program were $95^{\circ} \mathrm{C}$ for $10 \mathrm{~min}$ for $1 \mathrm{cycle}$, followed by 40 cycles of $95^{\circ} \mathrm{C}$ for $15 \mathrm{~s}$ and $60^{\circ} \mathrm{C}$ for $60 \mathrm{~s}$. This process was repeated for 40 cycles using the ABI-7500 (Applied Biosystems, Waltham, MA, USA). The expression levels were quantified relative to the values obtained for housekeeping genes ( $A C T B, B 2 M, G A P D H$, $H P R T 1$, and RPLP0). Data analyses were performed using webbased analysis software (http://pcrdataanalysis.sabiosciences. com/pcr/arrayanalysis.php).

\section{Statistical Analyses}

GraphPad Prism5 (San Diego, CA, USA) was used to analyze the clinical data. A $t$-test or two-way ANOVA followed by Tukey's post hoc test was used to determine significant differences between two or more groups, respectively. A $p$-value of less than 0.05 was considered significant.

\section{RESULTS}

\section{Isolation and Identification of Exosomes}

We assembled ascites or peritoneal washings from three ovarian cancer patients and three benign ovarian cyst patients. The isolated exosomes were identified under a transmission electron microscope at $80.0 \mathrm{kV}$ and ranged from 50 to $100 \mathrm{~nm}$ (Figure 1). The expression of the tetraspanin protein CD63 was detected by ELISA to determine the concentration of purified exosomes resuspended in PBS, i.e., $1 \times 10^{10}$ exosome particles per milliliters. The solutions were stored at $-80^{\circ} \mathrm{C}$ until use.

\section{Array Analysis}

A multiphase case-control study was used to examine differences in the mRNA expression levels of immunity-related genes among healthy PBLs treated with different exosomes. The PBLs were collected from five matched healthy individuals and were pooled to eliminate individual differences. A total of 84 key genes involved in mediating the communication between tumor cells and the cellular mediators of inflammation and immunity were screened using the Human Cancer Inflammation and Immunity Crosstalk $\mathrm{RT}^{2}$ Profiler PCR Array in PBLs stimulated by malignant and benign exosomes. Based on positive PCR controls and reverse transcription controls, this profile had high reproducibility and efficiency according to the RT ${ }^{2}$ Profiler PCR Array Data Analysis.

In terms of the sensitivity and accuracy, the cutoff fold change $\left(2^{-\Delta \Delta C t}\right)$ was set at greater than 5.0 or less than -4.0 . Genes that met the two criteria (average threshold cycle $<30$ and the foldchange cutoff between the malignant exosome group and benign exosome group) were selected for further qRT-PCR validation using lymphocytes from malignant ascites of EOC patients and from peritoneal washings of benign ovarian cyst patients. We found that 26 genes were overexpressed, whereas only two genes were downregulated in the malignant exosome group compared to the benign ascite exosome group (Figure 2; Table 1, $p<0.05$ ).
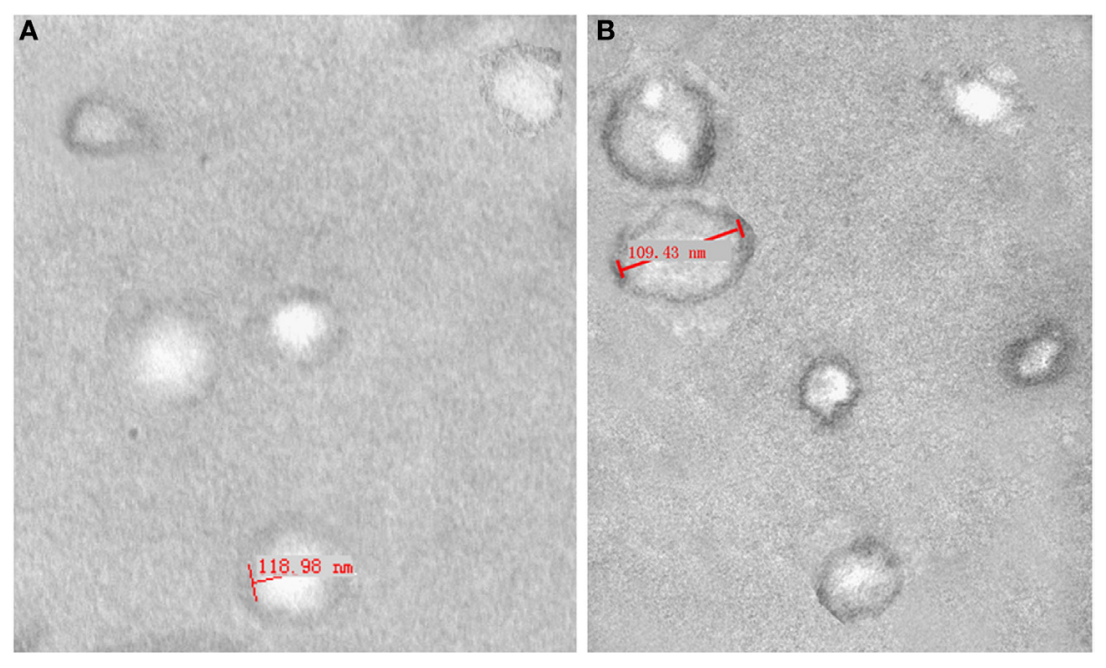

FIGURE 1 | Electron micrograph analyses of exosomes from peritoneal washings (A) and malignant ascites (B). Structural analysis of exosomes shows the presence of vesicles in the size range of $40-120 \mathrm{~nm}$. 


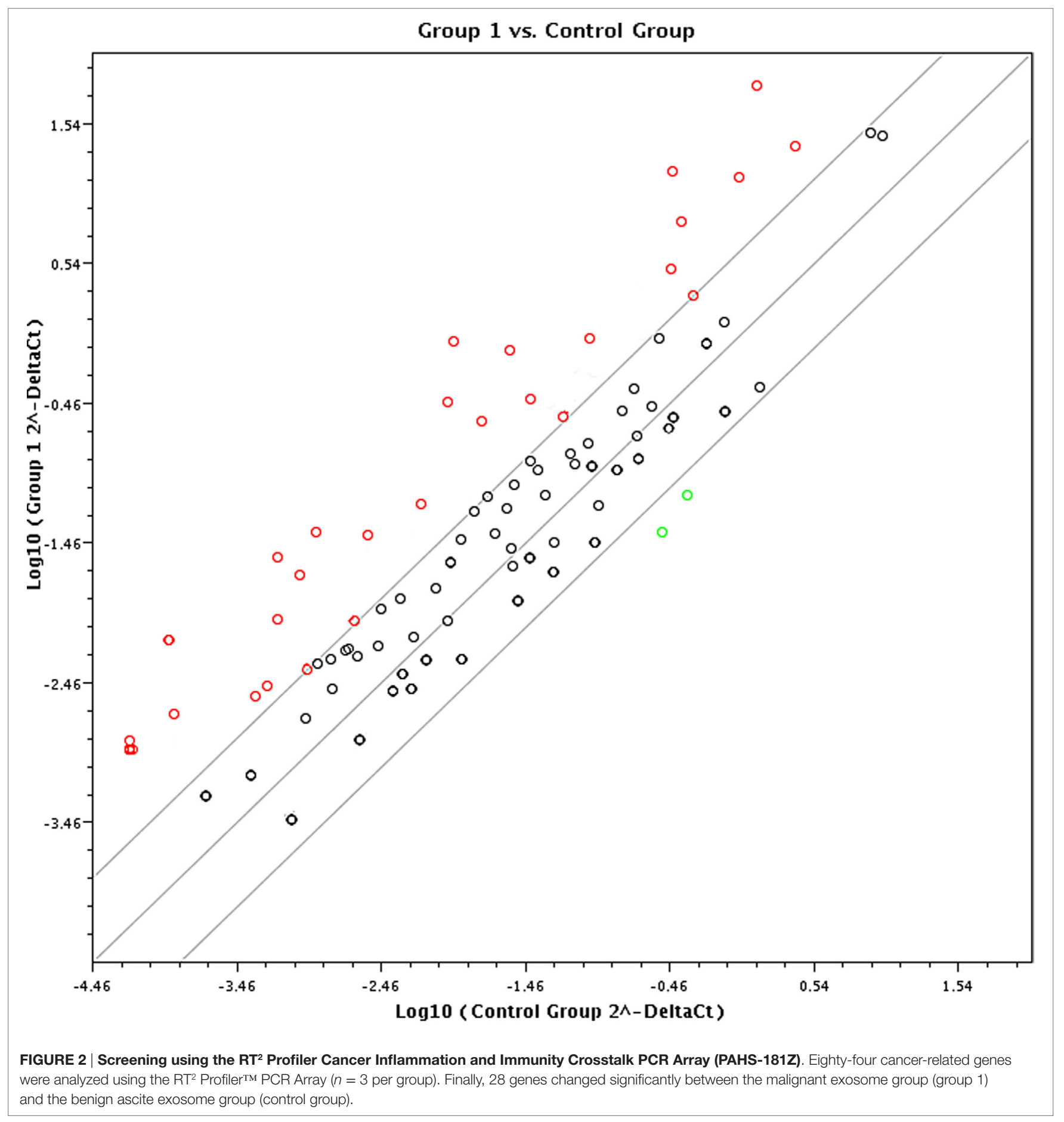

\section{Cancer Immunity Pathway Analysis}

The differentially expressed mRNAs in PBLs, which resulted from stimulation by malignant exosomes or benign exosomes, were mainly genes encoding for chemokines (C-C motif ligands and $\mathrm{C}-\mathrm{X}-\mathrm{C}$ motif ligands), interleukins, growth factors, and antigen presentation (Table 2). These overexpressed molecules were involved in immunostimulation, pro-inflammation, and immunosuppression in anticancer immunity. Acting as chemoattractants that guide cell migration, most chemokines (6/8 CC motif ligands and 5/7 CXC motif ligands) were more highly expressed after treatment with malignant exosomes than benign exosomes, suggesting that exosomes from cancer cells participate in immune surveillance, angiogenesis, and metastasis via the regulation of chemokine expression in PBLs.

In an analysis of the roles of the differentially expressed genes in signal transduction, we found that malignant exosomes were 
TABLE 1 | Profiler PCR array results for exosome-treated peripheral blood lymphocytes (malignant group vs. benign group).

\begin{tabular}{|c|c|c|c|c|c|}
\hline Refseq & Gene & $\begin{array}{l}\text { Fold } \\
\text { change }\end{array}$ & $p$-Value & Description & Gname \\
\hline NM_002982 & CCL2 & 10.613 & 0.026 & Chemokine (C-C motif) ligand 2 & $\begin{array}{l}\text { GDCF-2/HC11/HSMCR30/MCAF/MCP-1/MCP1/ } \\
\text { SCYA2/SMC-CF }\end{array}$ \\
\hline NM_002984 & CCL4 & 46.842 & 0.047 & Chemokine (C-C motif) ligand 4 & $\begin{array}{l}\text { ACT2/AT744.1/G-26/HC21/LAG-1/LAG1/MIP-1-beta/ } \\
\text { MIP1B/MIP1B1/SCYA2/SCYA4 }\end{array}$ \\
\hline NM_002985 & CCL5 & 9.331 & 0.031 & Chemokine (C-C motif) ligand 5 & D17S136E/RANTES/SCYA5/SIS-delta/SISd/TCP228/eoCP \\
\hline NM_002988 & CCL18 & 34.269 & 0.013 & Chemokine (C-C motif) ligand 18 & $\begin{array}{l}\text { AMAC-1/AMAC1/CKb7/DC-CK1/DCCK1/MIP-4/PARC/ } \\
\text { SCYA18 }\end{array}$ \\
\hline NM_004591 & CCL20 & 5.658 & 0.021 & Chemokine (C-C motif) ligand 20 & $\begin{array}{l}\text { CKb4/Exodus/LARC/MIP-3-alpha/MIP-3a/MIP3A/SCYA20/ } \\
\text { ST38 }\end{array}$ \\
\hline NM_148672 & CCL28 & 10.965 & 0.015 & Chemokine (C-C motif) ligand 28 & CCK1/MEC/SCYA28 \\
\hline NM_001511 & CXCL1 & 35.459 & 0.007 & Chemokine (C-X-C motif) ligand 1 & FSP/GRO1/GROa/MGSA/MGSA-a/NAP-3/SCYB1 \\
\hline NM_002089 & CXCL2 & 15.039 & 0.033 & Chemokine (C-X-C motif) ligand 2 & $\begin{array}{l}\text { CINC-2a/GRO2/GROb/MGSA-b/MIP-2a/MIP2/MIP2A } \\
\text { SCYB2 }\end{array}$ \\
\hline NM_002994 & CXCL5 & 6.084 & 0.015 & Chemokine (C-X-C motif) ligand 5 & ENA-78/SCYB5 \\
\hline NM_000584 & CXCL8 & 16.523 & 0.039 & Interleukin 8 & $\begin{array}{l}\text { GCP-1/GCP1/IL8/LECT/LUCT/LYNAP/MDNCF/MONAP/ } \\
\text { NAF/NAP-1/NAP1 }\end{array}$ \\
\hline NM_001565 & CXCL10 & 11.039 & 0.031 & Chemokine (C-X-C motif) ligand 10 & C7/IFI10/INP10/IP-10/SCYB10/crg-2/gIP-10/mob-1 \\
\hline NM_005409 & CXCL11 & 10.127 & 0.030 & Chemokine (C-X-C motif) ligand 11 & H174/I-TAC/IP-9/IP9/SCYB11/SCYB9B/b-R1 \\
\hline NM_000757 & CSF1 & 5.473 & 0.023 & Colony stimulating factor 1 (macrophage) & CSF-1/MCSF \\
\hline NM_014009 & FOXP3 & 7.014 & 0.047 & Forkhead box P3 & AIID/DIETER/IPEX/JM2/PIDX/XPID \\
\hline NM_002053 & GBP1 & 8.952 & 0.047 & Guanylate binding protein 1, interferon-inducible & - \\
\hline NM_004131 & GZMB & 13.799 & 0.032 & $\begin{array}{l}\text { Granzyme B (granzyme 2, cytotoxic } \\
\text { T-lymphocyte-associated serine esterase 1) }\end{array}$ & $\begin{array}{l}\text { CCPI/CGL-1/CGL1/CSP-B/CSPB/CTLA1/CTSGL1/ } \\
\text { HLP/SECT }\end{array}$ \\
\hline NM_000619 & IFNG & 43.678 & 0.010 & Interferon, gamma & $|F G / I F|$ \\
\hline NM_000576 & IL1B & 8.323 & 0.024 & Interleukin 1, beta & IL-1/IL1-BETA/IL1F2 \\
\hline NM_000586 & IL2 & 21.128 & 0.040 & Interleukin 2 & IL-2/TCGF/lymphokine \\
\hline NM_000600 & IL6 & 88.217 & 0.001 & Interleukin 6 (interferon, beta 2) & BSF2/HGF/HSF/IFNB2/IL-6 \\
\hline NM_000882 & IL12A & 10.592 & 0.010 & Interleukin 12A & CLMF/IL-12A/NFSK/NKSF1/P35 \\
\hline NM_000585 & IL15 & 31.130 & 0.012 & Interleukin 15 & $\mathrm{IL}-15$ \\
\hline NM_000247 & MICA & 13.941 & 0.031 & MHC class I polypeptide-related sequence A & MIC-A/PERB11.1 \\
\hline NM_000582 & SPP1 & 9.988 & 0.031 & Secreted phosphoprotein 1 & BNSP/BSPI/ETA-1/OPN \\
\hline NM_003150 & STAT3 & 8.592 & 0.033 & Signal transducer and activator of transcription 3 & ADMIO/APRF/HIES \\
\hline NM_003376 & VEGFA & 11.365 & 0.037 & Vascular endothelial growth factor A & MVCD1NEGFNPF \\
\hline NM_002116 & HLA-A & -5.914 & 0.034 & Major histocompatibility complex, class I, A & HLAA \\
\hline NM_005514 & HLA-B & -7.404 & 0.012 & Major histocompatibility complex, class I, B & AS/HLAB/SPDA1 \\
\hline
\end{tabular}

involved in the tumor immune process mainly via the interferon signaling pathway, STAT signaling pathway, and NF- $\mathrm{kB}$ signaling pathway (Table 3). To confirm this, signaling pathway activity was examined in lymphocytes from malignant ascites of six EOC patients and peritoneal washings of six benign ovarian cyst patients by western blotting (Figure 3). We observed no differences in the phosphorylation levels of IRF5/7/9 and STAT1/2/3/5/6 in the nucleus. However, the phosphorylation levels of IRF3 and p65 in the nuclei of lymphocytes from malignant ascites were higher than those from benign ascites, suggesting that the interferon and $\mathrm{NF}-\mathrm{\kappa B}$ signaling pathways are involved in the immune regulation of malignant exosomes.

\section{Validation of Lymphocyte Gene Expression in the Disease Microenvironment}

The 28 genes that were differentially expressed in normal PBLs treated with malignant or benign exosomes according to the array analysis were individually validated in lymphocytes from malignant ascites of 27 EOC patients or peritoneal washings of nine benign ovarian cyst patients by real-time PCR analysis. Regardless of clinicopathological characteristics, significant expression differences were observed, including 10 upregulated genes (CCL4/18, CXCL1/10/11, IFN , IL2/6/12A, VEGFA) and 1 downregulated gene (HLA-A) in lymphocytes from malignant ascites (Figure 4). 
TABLE 2 | Classification of differentially expressed genes according to their function.

\begin{tabular}{llc}
\hline Immune and inflammatory responses & Number/total \\
\hline Immunostimulatory factors & IFNG, IL2, IL12A, IL15 & $4 / 6$ \\
Immunosuppressive factors & CXCL5, VEGFA & $2 / 16$ \\
Pro-inflammatory genes & CCL2 (MCP-1), CCL20 & $9 / 15$ \\
& (MIP-3A), IFNG, IL1A, IL1B, & \\
& IL2, IL6, IL12A, VEGFA & \\
Enzymatic modulators of & GZMB & $1 / 6$ \\
immunity & & \\
Chemokines & & \\
C-C motif ligand & CCL2 (MCP-1), CCL4 (MIP-1B), & \\
& CCL5 (RANTES), CCL18 (PARC), & \\
C-X-C motif ligand & CCL20 (MIP-3A), CCL28 & \\
& CXCL1, CXCL2, CXCL5, CXCL10 & $5 / 7$ \\
Interleukins & (IP-10), CXCL11 (I-TAC, IP-9) & \\
Growth factors & IL1B, IL2, IL6, IL12A, IL15 & $5 / 13$ \\
Antigen presentation & CSF1 (MCSF), VEGFA & $2 / 8$ \\
\hline
\end{tabular}

TABLE 3 | Classification of differentially expressed genes according to signal transduction pathway.

\begin{tabular}{llc}
\hline $\begin{array}{l}\text { Signal transduction } \\
\text { pathway }\end{array}$ & Proportion \\
\hline $\begin{array}{lll}\text { Interferon signaling } \\
\text { Interferon-responsive }\end{array}$ & GBP1, IFNG, IL6 & $3 / 4$ \\
genes & CXL2 (MCP-1), CCL5 (RANTES), & $4 / 10$ \\
NFKB targets & CCL2 (MCP-1), CCL5 (RANTES), & \\
& CSF1 (MCSF), IFNG & $4 / 9$ \\
STAT targets & CCL2 (MCP-1), CCL4 (MIP-1B), CCL5 & $8 / 17$ \\
& (RANTES), CSF1 (MCSF), CXCL10 & \\
Transcription factors & (IP-10), CXCL11 (I-TAC, IP-9), IL1B, IL6 & \\
& FOXP3, STAT3 & $2 / 8$
\end{tabular}

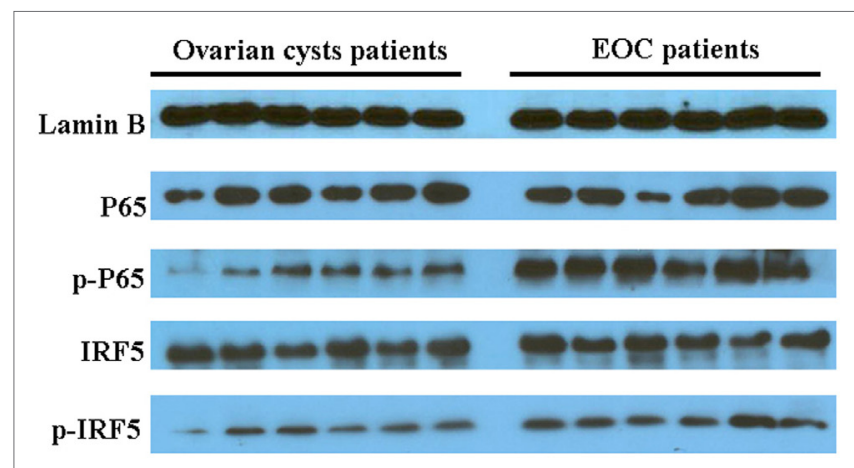

FIGURE 3 | Western blotting assays were used to analyze differences in signaling pathway activity between lymphocytes in malignant ascites of six epithelial ovarian cancer (EOC) patients and peritoneal washings of six benign ovarian cyst patients. The phosphorylation levels of IRF3 and p65 in the nucleus of lymphocytes from malignant ascites were higher than those from benign ascites.

\section{DISCUSSION}

Favorable accessibility for initial cytoreductive surgery and sensitivity to chemotherapy can increase the cure rates for ovarian cancer. However, the immune system of patients often have a substantial negative impact on the clinical course of the disease and curative effects $(27,28)$. Emerging evidence has indicated that antitumor immunity is often negated by immune suppression mechanisms in the tumor microenvironment, and cancerderived exosomes play a significant role in the recruitment and reprogramming of constituents in the tumor environment (29). Unlike healthy cells, cancer cells can secrete excess exosomes, and more importantly, they express distinct microRNAs with biological activity (30). Here, we used the commercially available $\mathrm{RT}^{2}$ Profiler ${ }^{\mathrm{TM}}$ Cancer Inflammation and Immunity Crosstalk PCR Array to identify useful molecular biomarkers and to clarify the pathway-based immunological system by evaluating differences in gene expression between PBLs treated with exosomes derived from benign and malignant ascites of ovarian cancer patients.

Exosomes are secreted by most cells to various bodily fluids, including the urine, blood, and cerebrospinal fluid, and actively participate in communication between cells via the transfer of bioactive proteins and nucleic acids (31). In this study, we used AIM-V-free serum to avoid interference caused by exosomes in fetal bovine serum. Currently, exosome research is limited by the lack of reliable and stable techniques to quantify and characterize exosomes. By detecting the CD63-specific antigen on exosomes, we standardized the stimulation of exosomes to fully explore differentially expressed genes in PBLs according to the biological properties and compositions of benign and malignant exosomes. Owing to limitations in available techniques, however, the purity of exosomes was not verified in this study. Accordingly, there may be trace amounts of other extracellular vesicles in the exosome samples.

Normalization strategies are critical for the success of mRNA expression studies. The combination of $A C T B, B 2 M, G A P D H$, HPRT1, and RPLP0 were used to normalize mRNA expression in this study based on the instructions provided by the array manufacturer. However, the microarray results suggested that the mRNA expression of two common internal reference genes were differentially expressed (GAPDH, 2.2-fold; $A C T B,-1.64$-fold). Accordingly, the fold change cutoff was set at greater than 5.0 or less than -4.0 to improve the sensitivity and accuracy.

High-quality exosomes isolation from complex biological fluids is a critical step for downstream functional analysis and therapeutic applications. To this end, numerous protocols and commercially available reagents have been designed to purify exosomes from various samples. However, as far as we know, to date no perfect method for exosomes isolation has been identified to isolate all exosomes from 30 to $150 \mathrm{~nm}$ without any impurities. Our study is also limited by the methods used in this study that may also precipitate non-exosome debris in urine samples (32). The gene expression of PBLs resulted from exosomes could be interfered by the debris. So, we eventually detected 26 upregulated genes and two downregulated genes in the malignant treatment group compared to the benign treatment group. These 28 genes were individually validated in the lymphocytes of ovarian cancer ascites and peritoneal washings of ovarian cysts by real-timePCR analysis. Eleven out of these 28 genes showed significant differential expression between cancer ascites and ovarian cysts (CCL4/18, CXCL1/10/11, IFN, IL2/6/12A, HLA-A, and VEGFA). 

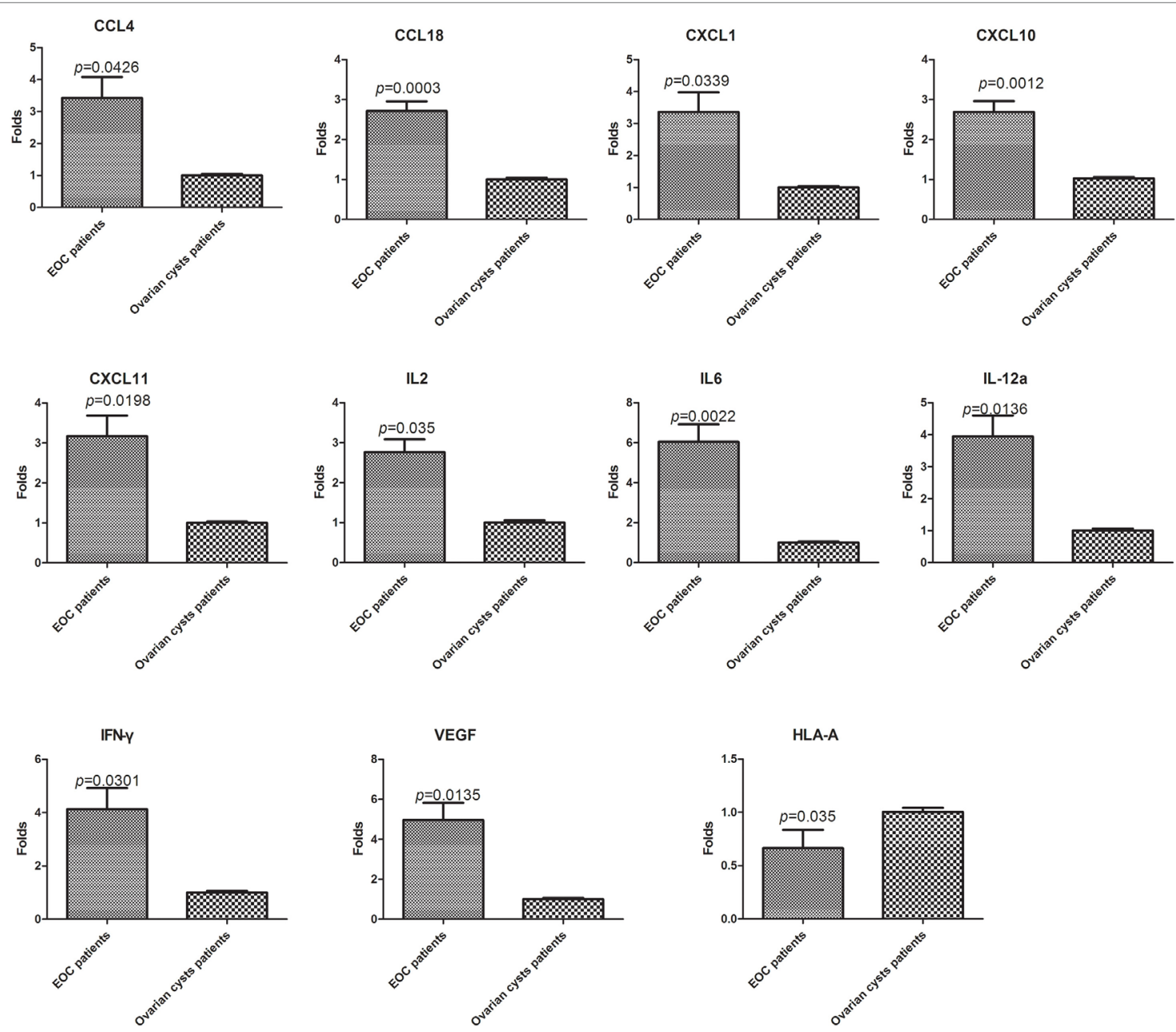

FIGURE 4 | Validation of the PCR screening results. The 28 genes identified using the RT ${ }^{2}$ ProfilerTM PCR array were validated using real-time PCR in lymphocytes from a larger sample of patients [27 epithelial ovarian cancer (EOC) patients and nine benign ovarian cyst patients]. Eleven genes showed significant differences $(p<0.05, t$-test).

Interestingly, there were significant increases in Foxp3 and IL10 expression, which are associated with immunosuppression in the tumor microenvironment.

Although the precise molecular mechanism and specific different constituents are unknown, these data suggest that malignant cells secreted exosomes in the tumor microenvironment to recruit lymphocytes not only to suppress antitumor immunity (IL6, Foxp3, and HLA-A/B) but also to enhance tumor invasion, tumor angiogenesis, and dissemination of proinflammatory cytokines (such as IL6 and VEGFA). More importantly, the immunity pathway analyses highlighted several genes with significant differences in mRNA expression in the $\mathrm{PCR}$ array, suggesting that the interferon and NF- $\mathrm{KB}$ signaling pathways are involved in the immune regulation of malignant exosomes. Owing to the complex nature of cancer and individual differences in the immune system, further studies with larger cohorts are warranted to confirm these results and determine key immunological biomarkers in the tumor microenvironment that may help develop better immunotherapeutic strategies.

\section{ETHICS STATEMENT}

The Ethics Committee of Nanjing Medical University. All research involving human participants was conducted with approval from the Ethics Committee of Nanjing Medical University. Written informed consent was obtained from all participants. None of the patients were treated with preoperative chemotherapy or radiation. No these additional considerations in the study.

\section{AUTHOR CONTRIBUTIONS}

YL and YY participated in the design of experiments, carried out the molecular analysis of cells, interpretation and 
analysis of in vitro and in vivo data, and helped to draft the manuscript. AX, XW, JX, and $\mathrm{SH}$ were involved in drafting the manuscript and participated in all experiments involving animals, including histological analyses. SZ have been involved in all aspects of the study, including experimental design, analysis and interpretation of data, and manuscript writing. All authors read and approved the final manuscript.

\section{REFERENCES}

1. Jemal A, Bray F, Center MM, Ferlay J, Ward E, Forman D. Global cancer statistics. CA Cancer J Clin (2011) 61:69-90. doi:10.3322/caac.20107

2. Wang B, Liu SZ, Zheng RS, Zhang F, Chen WQ, Sun XB. Time trends of ovarian cancer incidence in China. Asian Pac J Cancer Prev (2014) 15:191-3. doi:10.7314/APJCP.2014.15.1.191

3. Saad AF, Hu W, Sood AK. Microenvironment and pathogenesis of epithelial ovarian cancer. Horm Cancer (2010) 1:277-90. doi:10.1007/s12672-010-0054-2

4. Hansen JM, Coleman RL, Sood AK. Targeting the tumour microenvironment in ovarian cancer. Eur J Cancer (2016) 56:131-43. doi:10.1016/j. ejca.2015.12.016

5. Balkwill FR, Capasso M, Hagemann T. The tumor microenvironment at a glance. J Cell Sci (2012) 125:5591-6. doi:10.1242/jcs.116392

6. Kahlert C, Kalluri R. Exosomes in tumor microenvironment influence cancer progression and metastasis. J Mol Med (Berl) (2013) 91:431-7. doi:10.1007/ s00109-013-1020-6

7. Milane L, Singh A, Mattheolabakis G, Suresh M, Amiji MM. Exosome mediated communication within the tumor microenvironment. J Control Release (2015) 219:278-94. doi:10.1016/j.jconrel.2015.06.029

8. Au Yeung CL, Co NN, Tsuruga T, Yeung TL, Kwan SY, Leung CS, et al. Exosomal transfer of stroma-derived miR21 confers paclitaxel resistance in ovarian cancer cells through targeting APAF1. Nat Commun (2016) 29:11150. doi: $10.1038 /$ ncomms 11150

9. Tkach M, Théry C. Communication by extracellular vesicles: where we are and where we need to go. Cell (2016) 164:1226-32. doi:10.1016/j.cell.2016.01.043

10. Zomer A, Vendrig T, Hopmans ES, van Eijndhoven M, Middeldorp JM, Pegtel DM. Exosomes fit to deliver small RNA. Commun Integr Biol (2010) 3:447-50. doi:10.4161/cib.3.5.12339

11. Théry C, Zitvogel L, Amigorena S. Exosomes: composition, biogenesis and function. Nat Rev Immunol (2002) 2:569-79. doi:10.1038/nri855

12. Andre F, Schartz NE, Movassagh M, Flament C, Pautier P, Morice P, et al. Malignant effusions and immunogenic tumour-derived exosomes. Lancet (2002) 360:295-305. doi:10.1016/S0140-6736(02)09552-1

13. Braicu C, Tomuleasa C, Monroig P, Cucuianu A, Berindan-Neagoe I, Calin GA. Exosomes as divine messengers: are they the Hermes of modern molecular oncology? Cell Death Differ (2015) 22:34-45. doi:10.1038/cdd. 2014.130

14. Zhang X, Yuan X, Shi H, Wu L, Qian H, Xu W. Exosomes in cancer: small particle, big player. J Hematol Oncol (2015) 8:83. doi:10.1186/s13045-015-0181-x

15. Sangisetty SL, Miner TJ. Malignant ascites: a review of prognostic factors, pathophysiology and therapeutic measures. World J Gastrointest Surg (2012) 4:87-95. doi:10.4240/wjgs.v4.i4.87

16. Ayantunde AA, Parsons SL. Pattern and prognostic factors in patients with malignant ascites: a retrospective study. Ann Oncol (2007) 18:945-9. doi:10.1093/annonc/mdl499

17. Wu X, Zhi X, Liu M, Xie J, Zhao S. Elevated levels of dendritic cell-correlated miRNAs in ascites and sera of patients with ovarian cancer. Xi Bao Yu Fen Zi Mian Yi Xue Za Zhi (2015) 31:383-6. [Article in Chinese].

18. Liu M, Xie J, Zhao S. Detection of lymphocyte subsets in the peripheral blood of patients with malignant ovarian tumors. Xi Bao Yu Fen Zi Mian Yi Xue Za Zhi (2014) 30:627-34. [Article in Chinese].

19. Atanackovic D, Block A, de Weerth A, Faltz C, Hossfeld DK, Hegewisch-Becker S. Characterization of effusion-infiltrating $\mathrm{T}$ cells: benign versus malignant effusions. Clin Cancer Res (2004) 10:2600-8. doi:10.1158/1078-0432.CCR03-0239

\section{ACKNOWLEDGMENTS}

This work was supported by the National Natural Science Foundation of China (grant number: 81572557) State Key Laboratory of Reproductive Medicine (SKLRM-K201507) and the Medical Science and Technology Development Foundation of Nanjing (Department of Health, grant numbers: ZKX15029 and YKK16122).

20. Kim S, Kim B, Song YS. Ascites modulates cancer cell behavior, contributing to tumor heterogeneity in ovarian cancer. Cancer Sci (2016) 107(9):1173-8. doi: $10.1111 /$ cas. 12987

21. Peng P, Yan Y, Keng S. Exosomes in the ascites of ovarian cancer patients: origin and effects on anti-tumor immunity. Oncol Rep (2011) 25:749-62. doi:10.3892/or.2010.1119

22. Keller S, Sanderson MP, Stoeck A, Altevogt P. Exosomes: from biogenesis and secretion to biological function. Immunol Lett (2006) 107:102-8. doi:10.1016/j. imlet.2006.09.005

23. Royo F, Zuñiga-Garcia P, Sanchez-Mosquera P, Egia A, Perez A, Loizaga A, et al. Different EV enrichment methods suitable for clinical settings yield different subpopulations of urinary extracellular vesicles from human samples. J Extracell Vesicles (2016) 5:10. doi:10.3402/jev.v5.29497

24. Salomon C, Yee S, Scholz-Romero K, Kobayashi M, Vaswani K, Kvaskoff D, et al. Extravillous trophoblast cells-derived exosomes promote vascular smooth muscle cell migration. Front Pharmacol (2014) 5:175. doi:10.3389/ fphar.2014.00175

25. van der PolE, Coumans FA, Grootemaat AE, Gardiner C, Sargent IL, Harrison P, et al. Particle size distribution of exosomes and microvesicles determined by transmission electron microscopy, flow cytometry, nanoparticle tracking analysis, and resistive pulse sensing. J Thromb Haemost (2014) 12:1182-92. doi:10.1111/jth.12602

26. Punt S, Houwing-Duistermaat JJ, Schulkens IA, Thijssen VL, Osse EM, de Kroon CD, et al. Correlations between immune response and vascularization qRT-PCR gene expression clusters in squamous cervical cancer. $\mathrm{Mol}$ Cancer (2015) 14:71. doi:10.1186/s12943-015-0350-0

27. Gajewski TF, Schreiber H, Fu Y. Innate and adaptive immune cells in the tumor microenvironment. Nat Immunol (2013) 14:1014-22. doi:10.1038/ ni. 2703

28. Finn OJ. Immuno-oncology: understanding the function and dysfunction of the immune system in cancer. Ann Oncol (2012) 23(Suppl 8):viii6-9. doi:10.1093/annonc/mds256

29. Iero M, Valenti R, Huber V, Filipazzi P, Parmiani G, Fais S, et al. Tumourreleased exosomes and their implications in cancer immunity. Cell Death Differ (2008) 15:80-8. doi:10.1038/sj.cdd.4402237

30. Melo SA, Sugimoto H, O’Connell JT, Kato N, Villanueva A, Vidal A, et al. Cancer exosomes perform cell-independent microRNA biogenesis and promote tumorigenesis. Cancer Cell (2014) 26:707-21. doi:10.1016/j.ccell. 2014.09.005

31. Keller S, Ridinger J, Rupp AK, Janssen JWG, Altevogt P. Body fluid derived exosomes as a novel template for clinical diagnostics. J Transl Med (2011) 9:86. doi:10.1186/1479-5876-9-86

32. Oosthuyzen W, Sime NE, Ivy JR, Turtle EJ, Street JM, Pound J, et al. Quantification of human urinary exosomes by nanoparticle tracking analysis. J Physiol (2013) 591(23):5833-42. doi:10.1113/jphysiol.2013.264069

Conflict of Interest Statement: The authors declare that the research was conducted in the absence of any commercial or financial relationships that could be construed as a potential conflict of interest.

Copyright (C) 2017 Li, Yang, Xiong, Wu, Xie, Han and Zhao. This is an open-access article distributed under the terms of the Creative Commons Attribution License (CC $B Y)$. The use, distribution or reproduction in other forums is permitted, provided the original author(s) or licensor are credited and that the original publication in this journal is cited, in accordance with accepted academic practice. No use, distribution or reproduction is permitted which does not comply with these terms. 